FORMATION Formation emploi

Revue française de sciences sociales

123 | Juillet-Septembre 2013

Pêle-mêle

\title{
Les aides à domicile : un engagement dans la formation tributaire du mode d'exercice
}

Agency and consumer-directed Home care workers: unequal individual levels of commitment to continuing training

Haushalts- und Pflegehelfer/innen: Weiterbildungswille je nach Berufsausübung Los auxiliares domiciliarios : un compromiso con la formación tributario del modo de ejercicio

Michèle Mansuy et Rémy Marquier

\section{(2) OpenEdition}

Journals

Édition électronique

URL : http://journals.openedition.org/formationemploi/4043

DOI : 10.4000/formationemploi.4043

ISSN : 2107-0946

Éditeur

La Documentation française

Édition imprimée

Date de publication : 30 septembre 2013

Pagination : 45-65

ISSN : 0759-6340

Référence électronique

Michèle Mansuy et Rémy Marquier, « Les aides à domicile : un engagement dans la formation

tributaire du mode d'exercice », Formation emploi [En ligne], 123 | Juillet-Septembre 2013, mis en ligne le 07 octobre 2015, consulté le 10 décembre 2020. URL : http://journals.openedition.org/ formationemploi/4043; DOI : https://doi.org/10.4000/formationemploi.4043 


\title{
Les aides à domicile : un engagement dans la formation tributaire du mode d'exercice
}

\author{
MiCHĖLE MANSUY \\ Economiste, diplômée de l'ENSAE (Ecole nationale de la statistique et de l'administration \\ économique). Actuellement responsable des études statistiques à l'Office de coopération \\ économique pour la Méditerranée et l'Orient (OCEMO)
}

Rémy MARQUIER

Economiste, diplômé de l'ENSAE (Ecole nationale de la statistique et de l'administration économique). Chef du bureau des collectivités locales à la DREES (Direction de la Recherche, des Etudes, de l'Evaluation et des Statistiques)

Résumé

Les aides à domicile : un engagement dans la formation tributaire du mode d'exercice

Les aides à domicile aux personnes fragilisées s'engagent de façon inégale dans la formation continue. Le diplôme initial et l'âge expliquent en partie ces différences, mais à diplôme égal, l'appétence pour la formation continue n'est pas la même pour tous (toutes). Le mode d'exercice de la profession joue un rôle clé, le mode prestataire facilitant l'accès à la formation continue, surtout pour les moins diplômés. L'expérience professionnelle a également un impact important. Enfin, la VAE (validation des acquis de l'expérience) est mieux connue des aides à domicile que des autres employé(e)s non qualifié(e)s et presque le tiers d'entre elles envisagent de s'y engager.

Mots clés : Aide à domicile, formation professionnelle, Validation des acquis professionnels

Abstract

Agency and consumer-directed Home care workers: unequal individual levels of commitment to continuing training

Home care workers for the frail elderly or disabled people have unequal chances to participate in continuing training. Disparities are partly related to age and to the level of Education and Training achieved. However, other issues are involved. First, the service model (agencies/registries vs consumer directed) is a key factor, the agency model giving easier access to continuing training, especially at the lower levels of 
Education and Training. The Labour Market experience has a significant effect too. It is also shown that accreditation for prior work experience is better known by home care workers than by other (so-called) unskilled workers. Nearly one third of them intend to apply for it.

Keywords: home help, continuing training, validation of acquired skills

Journal of Economic Literature: M 54, J 24, L 84

Traduction : Auteurs

Les premières associations d'aide à domicile aux personnes âgées en perte d'autonomie ont été créées au début du XXème siècle sur le mode du bénévolat (Cerc, 2008 ; Laforge, 2003). Elles se sont développées après la seconde guerre mondiale et surtout après $1953^{1}$. C'est alors que l'aide à domicile salarié ${ }^{2}$ apparait à côté des bénévoles et des aidants familiaux. L'encouragement des pouvoirs publics au maintien à domicile des personnes âgées préconisé par le rapport Laroque (1962) et jamais démenti depuis - d’une part, et le vieillissement de la population, d'autre part, ont eu pour conséquence le développement rapide de ces emplois.

En parallèle, l'offre de services correspondante s'est structurée sous des formes variées : des établissements publics (les centres communaux ou intercommunaux d'action sociale CCAS et CIAS), des associations, et plus récemment des entreprises privées y côtoient l'emploi direct des intervenants. A cette diversité d'offre correspond une diversité de statuts, de négociations collectives et aussi de certifications professionnelles. Partie intégrante de la conception du métier, la formation de ces salariés s'organise à partir de 1971², pour l'aide ménagère (Ennuyer, 2009). Il faut attendre 1988 pour voir apparaître la première certification professionnelle, le CAFAD (certificat d'aptitude aux fonctions d'aide à domicile).

Dans la période récente, deux logiques ont contribué au très fort essor de l'effectif des aides à domicile (le nombre d'aides à domicile et d'aides ménagères a augmenté de plus de $6 \%$ en moyenne annuelle entre 2004 et 2008, Dares, 2011).

D'une part, la nécessité de faire face à l'augmentation de la population âgée dépendante et à l'exigence de qualité de service pour ces publics spécifiques : ainsi, la loi 2002-2 a inclus les services d'aide à domicile dans la catégorie des établissements sociaux et médicosociaux, ceux-ci étant de ce fait soumis à l'autorisation du conseil général. La loi 2005-841

1 Le décret 53-1186 du 29 novembre 1953 portant réforme des lois d'assistance marque le début de la professionnalisation de ces métiers.

2 La loi du 16 juillet 1971 sur la formation professionnelle continue fait obligation aux associations d'aides ménagères employant plus de dix salariés de cotiser à $0,8 \%$ sur les salaires des aides ménagères pour leur formation professionnelle. 
a complété ce dispositif en facilitant l'insertion des entreprises privées dans le secteur des services d'aide à domicile en instituant la procédure d'agrément qualité. Cet agrément, accordé par le préfet, après avis du président du conseil général, constitue une procédure plus légère que celle de l'autorisation. Les deux régimes, autorisation et agrément qualité, sont conditionnés à des exigences de qualité quant aux services rendus (part minimale d'aidants diplômés dans le total des aidants notamment).

D'autre part, plusieurs mesures ont concouru à solvabiliser la demande des personnes dépendantes en termes d'aide à domicile, répondant ainsi à la fois à une logique d'aide aux personnes les plus dépendantes et de soutien à l'emploi dans le secteur des aides à domicile : exonération des charges patronales pour l'aide aux personnes âgées de plus de 70 ans (loi Seguin, 1987), et surtout création de l'allocation personnalisée d'autonomie (APA) en 2002 ; celle-ci ayant permis aux personnes dépendantes de recevoir, dans le cadre d'un plan d'aide, des prestations d'aide à domicile en limitant leur contribution financière.

Parallèlement, le développement du secteur de l'aide à domicile a été fortement encouragé par les mesures visant plus largement le secteur des services à la personne qui l'englobe : crédits d'impôts et allègements de charges, création du Chèque emploi service (1993) puis $\mathrm{du}$ Chèque emploi service universel (2005).

Au total, entre 2002 et 2009, le nombre de bénéficiaires de l'APA à domicile, dont l'immense majorité consomme des heures d'aide, est passé de 301000 à 718 000, soit une augmentation de 139 \% (Bérardier, Clément, 2011). Les politiques locales en matière d'APA, différenciées d'un département à l'autre, influent par ailleurs sensiblement sur les perspectives de formation des aides à domicile (Devetter, Horn, Jany-Catrice, 2011).

Ces deux logiques, solidarité envers les personnes âgées dépendantes et soutien à la création d'emplois, font de l'aide à domicile une profession atypique : d'un côté, une volonté de répondre à de fortes exigences de qualité, impliquant de former le mieux possible les salariés aux conditions spécifiques du travail auprès de personnes fragiles; de l'autre côté, une volonté de croissance de l'emploi, permettant notamment aux personnes éloignées du marché du travail de retrouver un emploi ou d'opérer une reconversion professionnelle, aucun diplôme n'étant exigé pour exercer le métier. D’ailleurs, dans les nomenclatures professionnelles, les aides à domicile sont classés parmi les employés dits non qualifiés, ce qui amène à s'interroger sur la ou les conventions de professionnalisation du secteur (JanyCatrice, Puissant, Ribault, 2009).

A ceci s'ajoute le fait que les diplômes et formations spécifiques au secteur sont relativement récents : le CAFAD, déjà cité ,n'était ouvert qu'aux seules personnes déjà en emploi. Le diplôme d'État d'auxiliaire de vie sociale (DEAVS) lui succède en 2002 et est ouvert aux personnes n'ayant jamais travaillé ainsi qu’à la validation des acquis de l'expérience (VAE). Parallèlement, de nombreux diplômes ou titres professionnels sont en cohérence avec le métier : BEP (brevet d'études professionnelles) carrières sanitaires et sociales (succédant au BEP préparatoire aux carrières sanitaires et sociales en 1993) avec ou sans la 
mention complémentaire d'aide à domicile (2004), titre d'assistante de vie délivré par le ministère du Travail (2003), etc. Par ailleurs, outre les formations certifiantes, la formation continue est également une des composantes de l'exercice du métier, que ce soit pour l'apprentissage des gestes et postures ou pour la prise en charge de personnes souffrant de la maladie d'Alzheimer ou d'autres troubles apparentés.

Cet article tente de faire le point sur l'appropriation actuelle de la certification et de la formation continue par les aides à domicile. Il s'appuie pour cela sur l'enquête auprès des intervenants au domicile des personnes fragilisées réalisée par la DREES (Direction de la recherche, des études, de l'évaluation et des statistiques) mi-2008 (encadré 1).

\section{Encadré 1}

\section{L'enquête auprès des intervenants au domicile des personnes fragilisées}

L'enquête auprès des intervenants au domicile des personnes fragilisée (dite IAD) visait à dresser un état des lieux des conditions de travail des aides à domicile, de leur formation, des motivations qui les ont incités à exercer leur métier, et des incidences que le métier peut avoir sur leur vie personnelle. Elle a été réalisée d'avril à juillet 2008.

Cibler les aides à domicile n'est pas chose aisée, ce qui explique pourquoi, à notre connaissance, aucune enquête statistique n'a été menée auparavant sur l'ensemble du secteur (croisant les différents modes d'exercice) : en effet, deux bases de données sont disponibles pour disposer de leurs coordonnées. La première, fournie par l'Institut de retraite complémentaire des employés de maison (IRCEM), permet d'atteindre les intervenants exerçant auprès de particuliers employeurs (que ce soit en mode direct ou par voie mandataire). La seconde est fournie par I'Agence nationale des services à la personne (ANSP), et contient les coordonnées des structures faisant appel à des aides à domicile, par voie prestataire ou mandataire. Le protocole d'enquête a dû dépasser plusieurs difficultés (Marquier, 2010a) :

La base de I'ANSP ne comprend que les coordonnées des structures d'aide à domicile, qu'il a donc fallu interroger afin qu'elles fournissent les coordonnées d'un échantillon d'intervenants ; Les données de l'IRCEM permettent de disposer des coordonnées des intervenants dont l'employeur bénéficie d'exonérations au titre de l'APA ou s'il a plus de 70 ans (cette base de données ne donne pas la profession précise des personnes). Dans ce dernier cas cependant, l'employeur peut être parfaitement valide et l'intervenant ne peut être considéré comme travaillant auprès de personnes fragiles (sauf s'il travaille auprès d'autres personnes qui, elles, sont fragiles). Une première interrogation, téléphonique, a été effectuée afin de cibler plus précisément les aides à domicile au travers d'un questionnaire filtre. 5914 intervenants ont ainsi été interrogés par téléphone. Parmi eux, 3238 (54,7 \%) faisaient partie du champ de l'enquête ;

Enfin, les bases de données se recoupent partiellement : les intervenants travaillant en mode mandataire peuvent potentiellement être sélectionnés dans les deux bases. De même, un intervenant peut travailler sous plusieurs modes à la fois et faire partie des deux bases. II n'était pas possible a priori de dénombrer les intervenants concernés par ces recouvrements. Une technique statistique spécifique (le partage des poids - tenant compte des nombres de liens entre les bases de données de chaque individu sélectionné) a été utilisée pour pallier cette difficulté, en posant notamment des questions ciblées, relatives au(x) mode(s) d'exercice dans le questionnaire.

Au final, 2587 aides à domicile ont été interrogés en face à face par un enquêteur, via un questionnaire fermé, pendant une heure environ. 
Nous analyserons notamment le lien entre les diplômes et titres obtenus par les aides à domicile et le métier qu'elles exercent, sous le prisme du déclassement professionnel, l'ampleur de celui-ci variant fortement suivant la définition retenue, normative ou statistique. Une large part de l'analyse sera consacrée à l'accès à la formation continue et à la VAE selon le mode d'exercice des aides à domicile (prestataire, mandataire ou en emploi direct auprès de particuliers employeurs) ; celui-ci discriminant vraisemblablement l'implication dans la formation au même titre que le niveau de diplôme, le premier étant par ailleurs très corrélé au second. La dernière partie propose une catégorisation des trajectoires de formation des aides à domicile, mise en regard du mode d'exercice. Enfin, nous conclurons sur la difficile professionnalisation au travers de la formation, tant d'un point de vue qualitatif - grâce à la reconnaissance qu'une formation occasionnerait - que quantitatif.

\section{Les aides à domicile : une technicité mal reconnue et un accès limité à la formation}

\subsection{Moins de quatre aides à domicile sur dix ont un diplôme ou un titre en lien avec le métier}

Comme évoqué en introduction, plusieurs raisons, tant politiques (logique d'aide aux personnes fragiles, logique d'insertion) qu'historiques (création relativement récente des diplômes relatifs à l'aide à domicile) ont impliqué un fort essor de la profession d'aide à domicile, rendant délicate la professionnalisation - au sens de volume de certification des compétences - de l'ensemble, que ce soit pour les aides à domicile déjà en exercice ou pour les nouveaux arrivants dans le métier. Ainsi en 2008, sur 515000 aides à domicile, seuls $38 \%$ déclarent ${ }^{3}$ posséder un diplôme ou un titre en lien avec leur métier (tableau 1). Deux sur dix possèdent le DEAVS, diplôme le plus élevé à cette date dans la hiérarchie des métiers de l'aide à domicile pour les organismes privés à but non lucratif depuis l'accord de branche du 29 mars $2002^{4}$. Le BEP carrière sanitaire et sociale ${ }^{5}$ ou le BEPA services à la personne comptent à eux deux $9 \%$ des aides à domicile. En revanche, la mention complémentaire au BEP, de création récente, est marginale $(0,5 \%)$. A la diversité de

\footnotetext{
3 Le diplôme déclaré n’est pas toujours celui réellement possédé. Le simple fait d'avoir amorcé un cursus diplômant peut inciter certains à déclarer être titulaires du diplôme correspondant. Les nombres indiqués ici sont donc a priori des majorants.

4 Accord du 29 mars 2002 relatif aux emplois et aux rémunérations (agréé par arrêté du 31 janvier 2003), étendu par arrêté du 7 avril 2005.

5 La dernière session de ce diplôme a eu lieu en 2012. A partir de 2013, il est remplacé par le BEP ASSP " accompagnement, soins et services à la personne ", diplôme intermédiaire délivré en cours de bac pro en trois ans ASSP (premiers diplômés en 2014).
} 
titres professionnels possibles correspond aussi une inégalité d'équivalences pour obtenir le DEAVS (Doniol-Shaw, 2011).

Tableau 1

\section{Part des aides à domicile disposant d'un diplôme ou d'un titre en lien avec le métier (en\%)}

\begin{tabular}{|l|c|}
\hline Aucun diplôme du secteur sanitaire ou social & 62,1 \\
\hline DEAVS ou CAFAD & 21,2 \\
Autre diplôme du domaine sanitaire et social & 8,7 \\
BEP carrière sanitaire et sociale & 7,8 \\
Titre professionnel d'assistante de vie & 3,2 \\
BEP agricole services à la personne & 1,4 \\
Diplôme d'État de TISF ou travailleuse familiale & 0,8 \\
Mention complémentaire aide à domicile & 0,5 \\
\hline
\end{tabular}

Source : DREES - enquête IAD, 2008.

Sigles : DEAVS : diplôme d'État d'auxiliaire de vie sociale ; CAFAD : certificat d'aptitude aux fonctions d'aide à domicile ; TISF : technicien d'intervention sociale et familiale.

Remarque : la somme est supérieure à $100 \%$ car les aides à domicile peuvent déclarer plusieurs diplômes ou titres.

Pour autant, en moyenne, les aides à domicile ont arrêté pour la première fois leurs études à 17 ans. Cet âge de fin d'études varie en fonction de l'âge des intervenants : de 16 ans pour les salariés les plus âgés (55 ans et plus), à 19 ans pour les plus jeunes d'entre eux (moins de 35 ans). Le niveau d'études des plus jeunes est plus élevé : près de la moitié des moins de 35 ans ont au moins atteint le niveau du lycée, contre $20 \%$ des 55 ans et plus. De plus, pour ceux qui ont le niveau du BEP, la spécialité sanitaire et sociale est sensiblement plus fréquente parmi les plus jeunes.

Seuls $14 \%$ des aides à domicile n'ont pas dépassé le niveau de l'école primaire. Fréquemment, ils ont atteint le niveau BEP, sans forcément avoir obtenu de diplôme (47\%), principalement dans la spécialité sanitaire et sociale $(14 \%)$ ou en secrétariat (12\%). Par ailleurs, même si tous les diplômes relatifs à l'aide à domicile correspondent au niveau BEP ou CAP, près de deux aides à domicile sur dix ont amorcé un cycle préparatoire au baccalauréat. $7 \%$ l'ont obtenu sans aller plus loin, et $6 \%$ ont même entamé des études supérieures. Une partie des aides à domicile a donc un niveau de diplôme supérieur à celui requis pour l'emploi occupé, ce qui caractérise le déclassement professionnel. Cependant, la part des aides à domicile déclassés diffère selon que l'on prend ce terme dans un sens normatif (titulaires d'un diplôme donnant accès à des positions qualifiées, intermédiaires ou cadres, au sens de la norme sociale en vigueur ; Giret, Lemistre, 2004) ou dans le sens statistique, leur diplôme étant supérieur à celui le plus fréquemment possédé par ceux qui exercent cette profession (Giret, NauzeFichet, Tomasini, 2006). 
Selon la définition statistique du déclassement, la possession du CAP ou du BEP ou d'un autre diplôme du même niveau étant le cas le plus fréquent, $13 \%$ des aides à domicile (ceux qui ont le bac ou plus) seraient déclassés, mais beaucoup plus parmi les jeunes (31\% des moins de 35 ans). Dans le sens normatif, les titulaires d'un CAP ou d'un BEP ou d'un titre équivalent devraient normalement être classés qualifiés, alors que les nomenclatures rangent tous les aides à domicile parmi les employés non qualifiés (Amossé et Chardon, 2006 ; Burnod, Chenu, 2001) : ils s’ajoutent aux diplômés de niveau supérieur parmi les déclassés, dont la proportion, selon cette définition atteint $60 \%$. Le désajustement entre diplôme possédé et position socio-professionnelle occupée par les aides à domicile tient donc surtout à ce que les qualités professionnelles propres aux aides à domicile sont insuffisamment formalisées et reconnues, ce qui conduit à les englober dans l'ensemble des employés réputés non qualifiés.

\subsection{Une absence de technicité formalisée qui freine la professionnalisation}

La reconnaissance de la qualification professionnelle des aides à domicile, gage de la qualité du service rendu, ne semble donc pas aller de soi. En effet, les compétences nécessaires aux aides à domicile : savoir-faire ménager, "prendre soin ${ }^{6}$ et qualités relationnelles, sont souvent considérées comme naturellement attachées au genre féminin et non explicitées, alors que la définition du métier et la nature du service rendu diffèrent sensiblement d'un aide à domicile à l'autre, avec des degrés de satisfactions très inégaux des personnes âgées et de leurs familles (Avril, 2003). Ceci rend plus complexe la professionnalisation de ces métiers, comme observé dès 1998 par Causse, Fournier et Labruyère. En effet, les compétences spécifiques aux aides à domicile sont très mal reconnues, l'absence de technicité formalisée constituant un obstacle à la professionnalisation. Les compétences techniques sont d'ailleurs rarement évoquées par les clients, qui jugent davantage la qualité de la prestation des intervenantes à l'aune de leur comportement ou de leur capacité d'adaptation à des horaires atypiques et irréguliers (Fourcade, Ourtau, 2004). Rien d'étonnant alors à ce que ces compétences techniques soient peu reconnues. Les aides à domicile sont alors comptabilisés parmi les employés réputés non qualifiés, et une majorité d'entre eux sont déclassés, au sens normatif de ce terme. Leurs conditions d'emploi se démarquent d'ailleurs assez peu de ceux des autres employés dits non qualifiés, ensemble dont la définition est délicate, la frontière avec les emplois qualifiés étant très floue (Rose, 2012) : faibles salaires, temps partiel contraint fréquent, pénibilité. Seul un degré d'autonomie plus important les en distingue.

6 En anglais, le care. 


\section{Graphique 1 : accès à la formation professionnelle continue en fonction du niveau d'études et du mode d'exercice}

\begin{tabular}{|c|c|c|c|}
\hline $\begin{array}{l}\text { Jamais } \\
\text { de formation }\end{array}$ & $\begin{array}{l}\text { Jamais, } \\
\text { mais c'est prévu }\end{array}$ & $\begin{array}{l}\text { Formation ancienne } \\
\text { (plus de } 3 \text { ans) }\end{array}$ & $\begin{array}{l}\text { Formation récente } \\
\text { ( } 3 \text { ans ou moins) }\end{array}$ \\
\hline
\end{tabular}

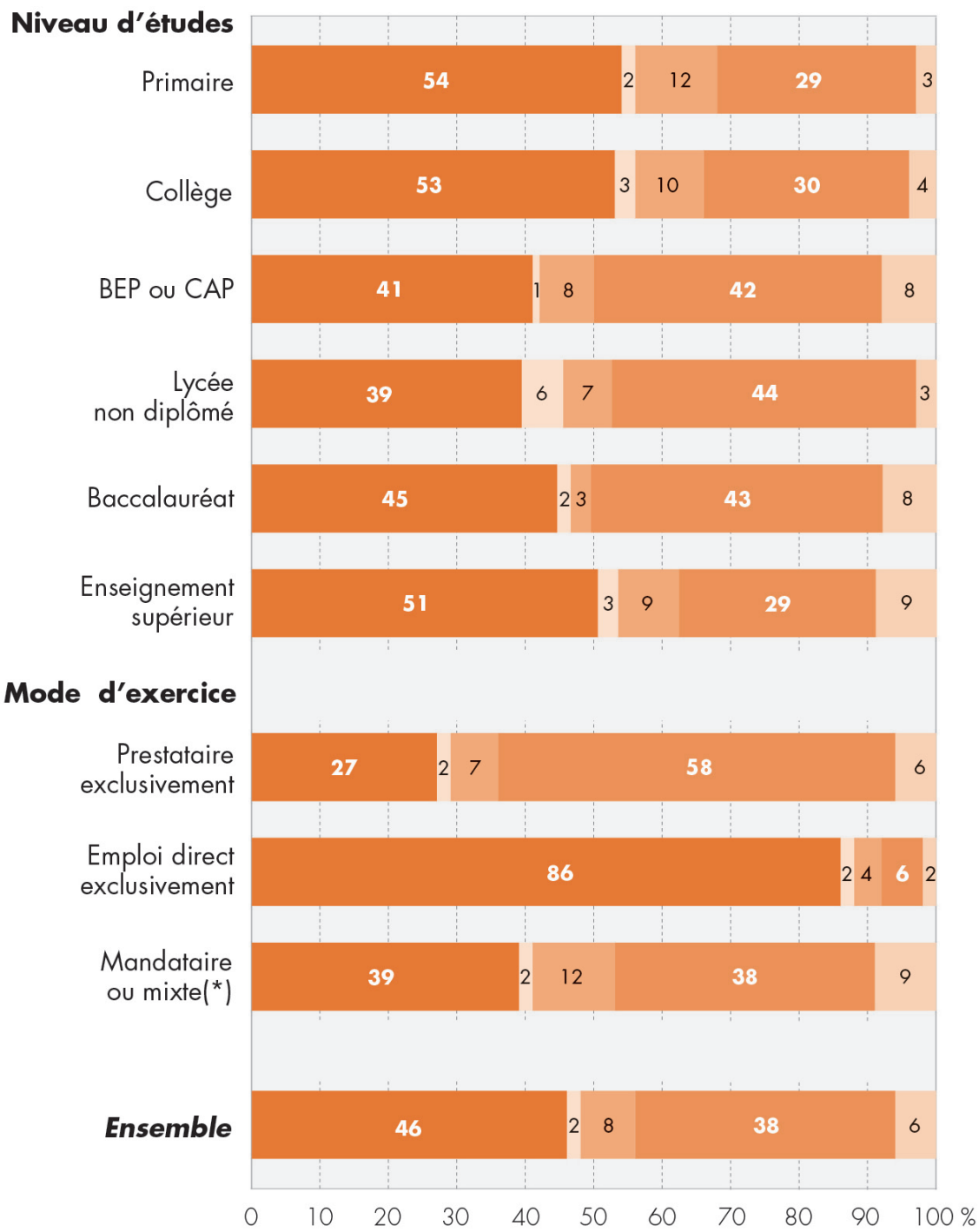

Source : DREES - enquête IAD, 2008

* Exerçant au moins deux des modes suivants : emploi prestataire, emploi mandataire ou emploi direct.

BEP : brevet d'études professionnelles ; CAP : certificat d'aptitude professionnelle. Lecture : 53,7 \% des aides à domicile qui n'ont pas été au-delà du primaire n'ont jamais suivi de formation professionnelle continue. 


\subsection{Les aides à domicile accèdent peu à la formation professionnelle continue}

Près de $46 \%$ des aides à domicile n'ont pas accédé à la formation continue et n'ont pas de projet précis dans ce sens (graphique 1). Cette proportion est encore plus forte parmi ceux dont le niveau d'études est le plus élevé (51 \% parmi les diplômés du supérieur), mais aussi parmi ceux dont le niveau est le plus faible (53\% de ceux qui n'ont pas dépassé le niveau du collège). Le moindre engagement des plus diplômés dans la formation pourrait apparaître comme une conséquence logique de leur situation de déclassement, au sens évoqué plus haut. Nous verrons par la suite qu'au niveau individuel, ce n'est pas systématiquement le cas.

La spécialité de formation a également une influence importante : les intervenants ayant un diplôme en relation avec le métier d'aide à domicile se forment davantage. Un tiers seulement de ceux qui ont atteint le niveau BEP du secteur sanitaire et social n’ont jamais été formés et n’ont pas de formation prévue. La propension à se former est d'autant plus grande que le diplôme obtenu a un lien étroit avec le métier d'aide à domicile : $36 \%$ des titulaires d'un BEP services n'ont pas eu accès à la formation, c'est le cas de $28 \%$ des détenteurs du titre d'assistante de vie et de $20 \%$ des titulaires du DEAVS, probablement du fait d'une volonté de reconnaissance technique plus importante, renforcée par le mode d'exercice : en effet, les titulaires de diplômes ou titres en lien avec le métier d'aide à domicile se retrouvent principalement dans les organismes prestataires (Marquier, 2010b).

On ne dispose pas de données directement comparables entre les aides à domicile et les autres employés dits non qualifiés. On sait cependant que ces derniers accèdent moins à la formation que l'ensemble des employés, eux-mêmes se formant moins que les cadres (respectivement $27 \%, 36 \%$ et $57 \%$ d'entre eux ont accédé à la formation continue dans les douze derniers mois ; source : enquête complémentaire à l'enquête emploi 2006 portant sur la formation continue ; INSEE, calculs Céreq). Dans chacune des catégories, les femmes accèdent moins à la formation que les hommes ${ }^{7}$. En effet, ce sont surtout les femmes qui doivent concilier les contraintes familiales avec les contraintes professionnelles. Elles se réorganisent plus souvent que les hommes pour suivre une formation (Fournier, 2009).

7 Il est utile de rappeler à ce titre que la profession d'aide à domicile est constituée à $98 \%$ de femmes. 


\section{Le mode d'exercice influe sur l'accès à la formation continue des aides à domicile}

\section{1 Être employé chez un prestataire favorise l'accès à la formation continue}

Le mode d'exercice de la profession (encadré 2) joue un rôle fort : 86 \% des aides à domicile en emploi direct n'ont jamais été formés, contre seulement $27 \%$ de ceux exerçant en mode prestataire.

Ces différences sont en partie liées à un moindre engagement des premiers dans la profession. En effet, les aides à domicile en emploi direct ont en majorité des durées de travail courtes ou très courtes (39\% d'entre eux travaillent moins de 3 heures par jour, et $66 \%$ moins de 7 heures ; contre $8 \%$ de ceux qui travaillent en mode prestataire et $28 \%$ de ceux qui exercent en mode mandataire ou mixte) (Barrois, Devetter, 2012). De plus, les réorganisations à mettre en place pour suivre une formation sont beaucoup plus importantes pour les intervenants en emploi direct, qui doivent gérer leurs relations d'emploi avec plusieurs employeurs particuliers, que pour les salariés en mode prestataire. Le cadre plus structuré des modes mandataire et surtout prestataire permet certainement d'organiser plus facilement des remplacements auprès des différents bénéficiaires de l'aide à domicile en formation et favorise l'accès à l'information des salariés sur les possibilités de formation professionnelle continue. D'autre part, les aides à domicile en emploi direct ont moins de velléités à la formation continue que les autres ( $c f$. infra).

Neuf fois sur dix, la formation suivie vise à améliorer les compétences. Le souhait d'évoluer vers un autre métier (4\% des cas) ou d'améliorer son salaire $(3,5 \%)$ viennent ensuite. D'ailleurs, les formations de courte durée dominent nettement : la dernière formation suivie a duré moins d'une semaine dans presque $60 \%$ des cas. Pour près de $24 \%$ des intervenants concernés, sa durée était comprise entre une semaine et un mois. Enfin, pour $18 \%$ des personnes concernées, la formation a duré plus d'un mois ${ }^{8}$. Quel que soit le mode d'exercice, les formations aux gestes professionnels, à la maladie d'Alzheimer et aux soins palliatifs sont les plus fréquemment suivies (tableau 2).

8 Les conventions suivantes ont été retenues : 7 heures = un équivalent jour ; 5 jours = un équivalent-semaine, 4 semaines = un équivalent-mois. 


\section{Encadré 2}

\section{Les modes d'exercice du métier d'aide à domicile}

Trois modes d'exercice sont possibles pour le métier d'aide à domicile :

- le mode prestataire : I'aide à domicile est salarié d'une structure d'aide à domicile. Le bénéficiaire de l'aide paie alors la prestation au service, qui rémunère le salarié ;

- le mode mandataire : le bénéficiaire de l'aide est lui-même l'employeur, mais une structure d'aide à domicile sert d'intermédiaire entre celui-ci et l'intervenant, notamment pour la mise en relation et les démarches administratives ;

- l'emploi direct : le bénéficiaire de l'aide est l'employeur et assume toutes les tâches administratives. II n'y a pas de structure intermédiaire.

Un même aide à domicile peut travailler sous différents modes, par exemple en étant à la fois salarié d'une structure prestataire pour une partie de ses heures de travail, et employé directement par un particulier en complément. Il est également possible de travailler sous les statuts de prestataire et de mandataire au sein d'une même structure.

L'enquête IAD ne permet pas d'isoler précisément le mode mandataire, ni de connaître les cumuls sous différents modes d'emploi. Elle permet toutefois de cibler les salariés travaillant exclusivement en mode prestataire (37\% des aides à domicile) et ceux travaillant exclusivement en emploi direct (24\%). Les autres aides à domicile travaillent soit en mode mandataire, soit en cumulant différents modes.

\section{Tableau 2}

\section{Contenu de la dernière formation suivie selon le mode d'exercice (en \%)}

\begin{tabular}{|l|c|c|c|c|}
\cline { 2 - 4 } \multicolumn{1}{c|}{} & $\begin{array}{c}\text { Salariés } \\
\text { d'organismes } \\
\text { prestataires }\end{array}$ & $\begin{array}{c}\text { Salariés en } \\
\text { emploi direct }\end{array}$ & $\begin{array}{c}\text { Salariés en mode } \\
\text { mandataire ou } \\
\text { mixtes ( *) }\end{array}$ & Ensemble \\
\hline Gestes et soins & 17 & 18 & 19 & 18 \\
\hline Maladie d'Alzheimer & 16 & 21 & 17 & 17 \\
\hline Soins palliatifs & 13 & 6 & 8 & 8 \\
\hline Relationnel & 7 & 11 & 10 & 7 \\
\hline Premiers secours & 8 & 6 & 5 & 5 \\
\hline Hygiène & 4 & 7 & 4 & 4 \\
\hline Manipulation d'appareils & 5 & 4 & 5 & 4 \\
\hline Diététique & 3 & 4 & 3 & 1 \\
\hline Prévention de la maltraitance & 4 & 3 & 1 & 1 \\
\hline Maladie de Parkinson & 2 & 0 & 0 & 0 \\
\hline Tutorat & 1 & 0 & 0 & 20 \\
\hline Gestion des conflits & 1 & 0 & 100 & 100 \\
\hline Autre & 19 & 20 & 5 & 5 \\
\hline Total & 100 & 100 & 7 & 5 \\
\hline
\end{tabular}

$\left(^{*}\right)$ Exerçant au moins deux des modes suivants : emploi prestataire, emploi mandataire ou emploi direct. Lecture : pour $17 \%$ des aides à domicile qui ont suivi au moins une formation professionnelle continue, la dernière de ces formations était consacrée aux gestes et soins.

Source : DREES - enquête IAD, 2008. 
Quant au souhait d'une prochaine formation, des différences importantes sont observées également en fonction du mode d'exercice des intervenants à domicile : ce souhait est exprimé par $74 \%$ de ceux qui exercent en mode prestataire, $62 \%$ de ceux qui exercent en mode mandataire ou mixte, et $31 \%$ de ceux qui exercent en emploi direct. Ceux qui n’ont jamais été formés et ceux qui l'ont été il y a plus de trois ans expriment moins souvent que l'ensemble le souhait d'une formation future (à peine plus de $40 \%$ sont dans ce cas, contre $59 \%$ de l'ensemble). Ce résultat rejoint celui mis en évidence par Fournier (2006) : ceux qui ont déjà été formés expriment plus fréquemment des besoins futurs. En effet, le souhait de se former dépend à la fois de l'apport espéré par l'intéressé, et de la probabilité d'aboutir qu'aurait, selon lui, sa demande éventuelle. Ces deux anticipations pousseraient ceux qui ont été formés antérieurement à formuler plus souvent que l'ensemble des demandes futures.

Comme pour l'ensemble des salariés, le souhait de se former est moins fréquent pour ceux dont le bagage scolaire est modeste : à peine plus du tiers des aides à domicile de niveau primaire, et $44 \%$ de ceux qui ont un niveau collège aspirent à se former. La propension à se former augmente ensuite avec le niveau d'études, puis diminue à nouveau. Il n'en reste pas moins que près de la moitié de ces aides à domicile les plus diplômés désirent se former dans le futur.

\subsection{Une plus grande implication dans la VAE aussi en mode prestataire}

Une majorité d'aides à domicile $(60 \%)$ sait que les diplômes peuvent être acquis par validation des acquis de l'expérience (VAE). Cette connaissance paraît beaucoup mieux diffusée que parmi l'ensemble des employés dits non qualifiés (seuls $35 \%$ de ces derniers connaissent la VAE ; source : Enquête Formation Continue 2006, INSEE, calculs Céreq ). La VAE est pourtant moins connue de ceux qui exercent en emploi direct (34\%, soit une proportion voisine de l'ensemble des employés non qualifiés). Les salariés travaillant en mode prestataire sont mieux informés sur la VAE que ceux qui exercent en mode mandataire ou mixte $(72 \%$ et $64 \%$ d'entre eux respectivement connaissent la VAE) (graphique 2). L'information sur la VAE apparaît corrélée au niveau de diplôme de l'aide à domicile : cette possibilité est plus souvent ignorée par ceux qui n'ont qu'un niveau primaire (deux fois sur trois) ou collège (une fois sur deux), que par ceux qui ont un niveau lycée ou supérieur (un peu plus de $20 \%$ de non-informés).

Presque $30 \%$ des aides à domicile enquêtés déclarent être déjà engagés dans un parcours de VAE ou être prêts à la faire. La première motivation de ceux qui s'engagent dans la VAE est le souhait d'obtenir un diplôme officiel du métier. Le souhait de changer de métier intervient très peu ( $2 \%$ des aides à domicile le citent). Cependant, les aides à domicile qui possèdent le bac ou un diplôme supérieur déclarent plus que les autres s'investir dans la VAE pour évoluer dans le métier ou en changer. 
Formation continue et VAE ne s'opposent pas, bien au contraire. En effet, c'est parmi les intervenants à domicile qui ont une formation prévue, en cours ou récente", que la VAE est la mieux connue (80\% d'entre eux déclarent en être informés) et la plus utilisée : $43 \%$ d'entre eux déclarent être engagés dans un parcours VAE ou en avoir l'intention.

\section{Graphique 2}

\section{Accès à la formation professionnelle continue en fonction du niveau d'études et du mode d'exercice}

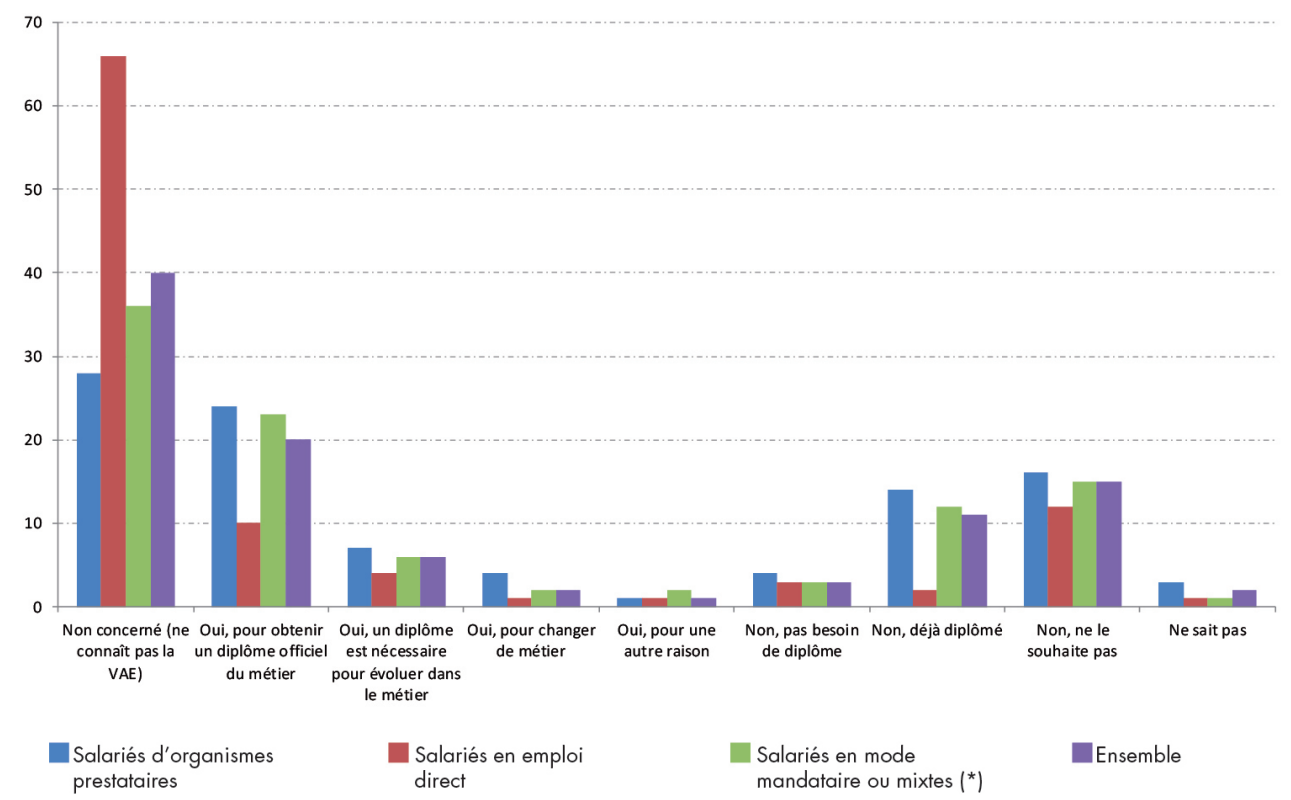

$\left(^{*}\right)$ Salariés en mode mandataire ou mixtes : Appartenant à au moins deux des modes d'exercice suivants : emploi prestataire, emploi mandataire ou emploi direct.

Lecture : $24 \%$ des aides à domicile salariés d'un organisme prestataire sont engagés ou prêts à s'engager dans un processus de VAE pour obtenir un diplôme officiel du métier.

Source : DREES - enquête IAD, 2008.

9 Au cours des trois dernières années. 


\section{Des profils de formations divers pour les aides à domicile}

On le voit, les profils de formation des aides à domicile sont divers, et comme pour l'ensemble des salariés, la formation continue semble relativement liée au niveau de diplôme et de formation.

\subsection{Sept profils diversement engagés dans la formation}

Des profils types des aides à domicile suivant la ou les formations suivies ou à venir peuvent être mis en exergue : une analyse des correspondances multiples (ACM), suivie d'une classification par la méthode des centres mobiles ${ }^{10}$ a permis de dégager sept profils, ceux-ci pouvant également être agrégés en quatre groupes distincts (graphique 3) : les aides à domicile peu impliqués dans le parcours de formation, les aides à domicile sans diplôme ou peu spécialisés mais très impliqués dans la formation continue, les aides à domicile très spécialisés et impliqués dans la formation continue, les aides à domicile ayant un niveau d'études relativement élevé, et qui désirent se former ultérieurement. Ces profils constituent des idéaux-types et ne s'appliquent pas individuellement à l'ensemble des membres des groupes ainsi constitués. Un profil est déterminé par les caractéristiques individuelles les plus souvent présentes, la norme de référence étant la moyenne de l'échantillon total.

Groupe 1 : les aides à domicile peu impliqués dans le parcours de formation $(39 \%$ des aides à domicile). Ce groupe se décompose en deux profils types, selon que la formation initiale est spécialisée ou non :

Profil 1 (20\% des aides à domicile) : les aides à domicile disposant d'un faible niveau d'études (niveau primaire pour $45 \%$ d'entre eux, niveau collège pour $44 \%$ ), sans spécialisation, et qui ne peuvent ou ne veulent pas suivre de formation continue : $60 \%$ pensent qu'ils n'ont pas accès à la formation continue, $76 \%$ ne veulent pas en suivre. De plus la plupart ignorent ce qu'est la VAE (84\%). Ces personnes ont par ailleurs arrêté pour la première fois leur scolarité très tôt (avant 17 ans);

Profil 2 (19\% des aides à domicile) : les aides à domicile à faible niveau d'études, mais avec une spécialisation, sans rapport avec l'aide à domicile toutefois : $75 \%$ ont un niveau BEP/CAP autre que dans le domaine sanitaire ou social. Comme pour le profil précédent, mais de façon moins marquée, la majorité pense ne pas pouvoir (52\%) ou vouloir $(60 \%)$ faire de formation continue. $62 \%$ ne connaissent pas la VAE.

10 Réalisée à partir des coordonnées des quatre premiers axes de l'ACM. Les variables actives ayant permis l'analyse des correspondantes multiples sont reprises pour décrire chaque classe ( $c f$. infra). 
Graphique 3 : typologie des profils de formation des aides à domicile

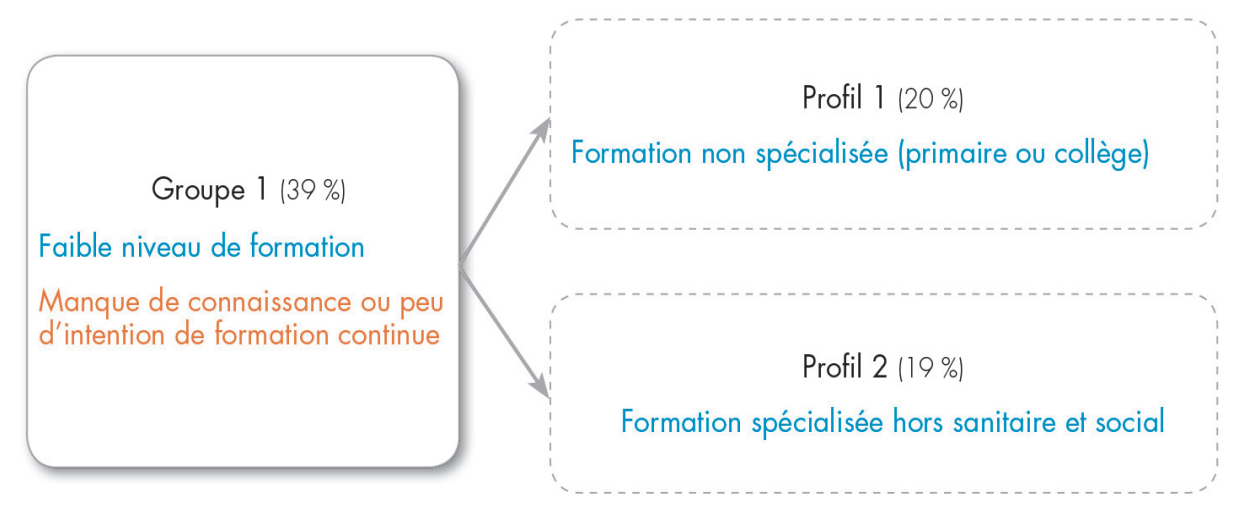

\section{Groupe $2(25 \%)$}

Faible niveau de formation

Forte implication dans le parcours de formation continue

\section{Profil $3(13 \%)$}

Formation non spécialisée (primaire ou collège)

Profil 4 (12\%)

Formation spécialisée hors sanitaire et social

\section{Groupe $3(14 \%)$}

Formation plus spécialisée

Forte volonté de se former
Profil 5 (14\%)

Formation spécialisé sanitaire et social DEAVS (Diplôme d'Etat Auxiliaire de vie sociale) ou CAFAD (Certificat d'aptitude aux fonctions d'aide à domicile)

\section{Groupe 4 (22\%)}

Niveau d'études plus élevé

Volonté de se former ultérieurement

\section{Profil $6(11 \%)$}

Niveau lycée ou supérieur

Forte implication dans la formation continue

\section{Profil $7(11 \%)$}

BEP sanitaire et social, baccalauréat ou supérieur Implication assez forte dans la formation continue 


\section{Groupe 2 : les aides à domicile sans diplôme ou titre spécialisé mais très impliqués dans la formation continue ( $25 \%$ des aides à domicile).}

Du point de vue du niveau de diplôme, ce groupe a globalement les mêmes caractéristiques que le précédent, mais est à l'opposé sur la formation continue ou les souhaits de formation à venir. Il se décompose lui aussi en deux profils types, selon que la formation initiale est spécialisée ou non :

Profil 3 (13\% des aides à domicile) : les aides à domicile à faible niveau d'études et sans spécialisation (31\% au niveau primaire, $39 \%$ au niveau collège, les trois quarts n'ayant aucun diplôme sanitaire ou social), mais ayant une forte volonté de se former ou d'évoluer : $60 \%$ ont suivi une formation continue récemment (dans les trois ans), et $21 \%$ ont déjà programmé la prochaine formation ;

Profil 4 (12\% des aides à domicile) : les aides à domicile à faible niveau d'études, mais avec une spécialisation hors sanitaire et sociale (68\% ont un BEP/CAP en dehors de cette spécialité), ou à niveau d'études intermédiaire (16\% ont été jusqu'au lycée sans obtenir le baccalauréat). Comme pour le profil précédent, l'implication dans la formation continue est relativement forte : $19 \%$ sont en cours de formation continue et $65 \%$ en ont suivi une dans les 3 ans passés. Par ailleurs, $52 \%$ sont engagés dans un processus de VAE pour obtenir le diplôme ad hoc ou l'envisagent.

Groupe 3 : les aides à domicile très spécialisés et impliqués dans la formation continue (14\% des aides à domicile). Ce groupe ne contient qu'un profil type :

Profil 5 (14\% des aides à domicile) : les aides à domicile très spécialisés dans leur métier (87 \% déclarent être titulaires du DEAVS ou du CAFAD, $10 \%$ du BEP carrière sanitaire et sociale avec ou sans la mention complémentaire d'aide à domicile, ou encore du BEPA service à la personne). De plus, $77 \%$ ont suivi récemment une formation continue (dans les 3 ans) et $65 \%$ désirent en faire une dans l'avenir.

Groupe 4 : les aides à domicile ayant un niveau d'études relativement élevé, et qui désirent se former ultérieurement (13\% des aides à domicile). Ce groupe comporte deux profils types :

Profil 6 (11\% des aides à domicile) : les aides à domicile ayant un niveau d'études supérieur à celui le plus souvent exigé pour l'exercice du métier : $23 \%$ ont été au lycée sans obtenir le baccalauréat, $24 \%$ ont obtenu au maximum le baccalauréat et $10 \%$ ont été jusqu'aux études supérieures. Ces aides à domicile sont par ailleurs impliqués dans la formation continue : $52 \%$ ont suivi une formation dans les trois dernières années et $19 \%$ sont en cours de formation. De même, $91 \%$ désirent suivre une formation dans le futur, celle-ci pouvant déjà être programmée. Ils affichent également des velléités pour la validation des acquis de l'expérience : 53 \% sont ou désirent s'engager dans un démarche de VAE afin d'obtenir le diplôme adéquat pour l'exercice de leur métier, et 17 \% pour évoluer dans ce métier ; 
Profil 7 (11\% des aides à domicile) : les aides à domicile ayant un niveau de formation suffisant pour exercer le métier (39\% sont titulaires du BEP carrière sanitaire et sociale avec ou sans la mention complémentaire d'aide à domicile, ou du BEPA service à la personne), ou supérieur à ce qui est habituellement exigé pour exercer le métier ( $22 \%$ sont allés jusqu'au bac, $32 \%$ ont fait des études supérieures). Ils affichent une certaine volonté de s'engager dans un processus de formation continue : si $71 \%$ n'en ont pas encore fait, ils sont $57 \%$ à désirer s'y engager dans le futur.

Ces quatre groupes sont cohérents avec les résultats des travaux de Charlon-Dubar et alii $(1990)^{11}$, selon lesquels quatre types de réaction face a l'offre de formation sont identifiables. Ainsi le groupe 1 (individus peu impliqués dans la formation continue) peut être associé aux individus exprimant un " refus " de formation (" les refusants ", le groupe 2 (individus peu qualifiés mais très engagés dans la formation continue) aux "retraducteurs ", c'est-a-dire ceux désirant accéder a une promotion sociale, le groupe 3 (individus très spécialisés et impliqués dans la formation continue) aux " adhérents " et le groupe 4 (individus plutôt diplômés et envisageant de se former ultérieurement) aux « hésitants».

Le mode d'exercice des aides à domicile influe sur leur profil de formation : ainsi les employés en mode prestataire sont plus souvent présents parmi les " retraducteurs » et les " adhérents" que ceux travaillant en emploi direct. A l'inverse, les salariés prestataires se retrouvent beaucoup moins parmi les « refusants».

\subsection{La formation des aides à domicile : un bilan en demi-teinte}

Au total, malgré la politique de professionnalisation mise en place par les pouvoirs publics, la formation des aides à domicile peut paraittre limitée : la plus grande partie d'entre eux ne dispose que d'un faible niveau de formation (près des deux tiers n'ont aucun diplôme du secteur sanitaire et social) et parmi eux, une assez forte proportion n'est que peu investie dans un processus de formation continue ou certifiante. Toutefois, la typologie des aides à domicile présentée ici montre une variété de situations rencontrées et d'attitudes envers la formation dans cette profession. Même si la proportion d'intervenants peu formés et sans véritable projet de formation future est importante (groupe 1 de la typologie précédente : individus peu impliqués dans la formation continue), il n'en reste pas moins qu'environ six aides à domicile sur dix sont impliqués dans un processus de formation continue, parfois très activement. Le mode d'exercice de la profession d'aide à domicile semble ici révélateur : ainsi, même parmi les aides à domicile avec le plus faible bagage de formation, le travail en mode prestataire semble favoriser l'accès et les velléités de formation continue (groupe 2 : individus peu qualifiés mais très engagés dans la formation continue). Si leur projet aboutit

11 Cité par notamment par Fournier (2006). 
à une certification, il peut leur permettre de se stabiliser dans l'emploi d'abord, de connaître une évolution dans le métier ensuite (Doniol-Shaw et alii, 2007). Les aides à domicile les mieux formés sont également ceux qui ont le plus d'appétence pour la formation continue (groupe 3 : individus très spécialisés et impliqués dans la formation continue), même si passé un certain seuil de niveau d'étude, celle-ci s'amenuise (groupe 4 : individus plutôt diplômés et envisageant de se former ultérieurement).

En termes de salaire horaire ou de conditions de travail, les gains apportés aux aides à domicile par la formation et la certification sont cependant limités. Le gain essentiel résiderait, pour ceux qui exercent en mode mandataire ou prestataire, dans l'accès aux « pseudos marchés internes » des structures qui les emploient, c'est-à-dire dans l'accès progressif, par l'expérience professionnelle et la certification des compétences acquises, à un portefeuille d'interventions mieux organisé permettant de se rapprocher du temps complet (Barrois, Devetter, 2012). Etre formé ou certifié leur permettrait un meilleur remplissage de leur planning hebdomadaire, jusqu'à une durée proche du temps complet, et donc de percevoir un salaire mensuel plus élevé. Mais cela au prix de contraintes fortes : des horaires décalés, une emprise du travail dans la journée importante en raison des transports et des temps de battement, une pénibilité du travail intensifiée.

Par ailleurs, les perspectives de promotion interne au métier sont restreintes. En effet, les postes de cadre ou de profession intermédiaire du secteur ne sont pas, sauf exception, pourvus par des intervenantes promues. De plus, l'utilité d'un encadrement de proximité fait débat et cette position n'est pas certifiée par un diplôme de niveau bac (Bouvier, Pelvillain, Santelmann, 2010). Se diriger vers le secteur de l'hébergement, voire changer complètement de domaine d'activité, semble être la seule voie d'évolution pour les diplômés.

On retrouve ici, dans les caractéristiques de la formation continue des aides à domicile, les résultats de Fournier $(2004$; 2006) pour l'ensemble des emplois non qualifiés, à ceci près que la porte de la formation continue peut rester ouverte pour les moins diplômés, en particulier pour ceux qui exercent dans un cadre structuré.

\section{Conclusion}

On peut au final s'interroger sur l'évolution quantitative des diplômés du secteur de l'aide à domicile. La croissance de la population des personnes fragilisées est particulièrement forte depuis une décennie et cette tendance devrait se poursuive suite à l'allongement de la durée de la vie et l'arrivée aux âges avancés de la génération du baby boom (Jolly, Liégey, Passet, 2012). Si le nombre d'aidants proches (famille, voisins...) ne devrait pas fondamentalement évoluer en proportion des personnes âgées dépendantes (Charpin, Tlili, 2011), le besoin quantitatif d'aides à domicile ne fait aucun doute dans le futur : on prévoit ainsi un besoin de recrutement de plus de 300000 professionnels entre 2010 et 2020, dont 
la moitié est due aux remplacements des départs et l'autre aux besoins démographiques (Lainé, Omalek, 2012).

D'un point de vue qualitatif, la volonté de professionnaliser le secteur de l'aide à domicile pour assurer un service de qualité - et donc pour partie de former les aides à domicile - semble largement partagée (Devetter et alii, 2008). Elle butte actuellement sur la faible reconnaissance de leur technicité spécifique (Fourcade, Ourteau, 2004), voire le déni de leurs compétences, en particulier pour celles qui n'exercent pas dans un cadre structuré et sont largement exclues de la formation et de la VAE. De plus, la reconnaissance de la qualité du service passe par celle de la qualité de la prestation des intervenantes, qui revêt des formes multiples, impossibles à standardiser. Prendre en compte cette diversité est en soi un enjeu pour la professionnalisation des aides à domicile (Rose, 2012).

In fine, on ignore encore si le nombre d'aides à domicile diplômés ou certifiés sera suffisant, et si la reconnaissance de ces diplômes ou titres en termes de salaire et de conditions de travail sera suffisamment améliorée pour leur permettre de rester durablement dans la profession, afin de conserver le rapport actuel entre diplômés et non diplômés parmi les intervenants, voire de l'augmenter, les contraintes budgétaires fortes des collectivités entrant notamment en conflit avec cet objectif ${ }^{12}(\operatorname{Lada}, 2011)$.

\section{Bibliographie}

Amossé T. et Chardon O. (2006), "Les travailleurs non qualifiés : une nouvelle classe sociale ?", Économie et Statistique, n 393-394, pp. 203-228.

Avril C. (2003), "Les compétences féminines des aides à domicile ", in Charges de famille (Weber, Gojard, Gramain (eds)), pp. 187-207.

Barrois A. et Devetter F.-X. (2012), «Aides à domicile : un régime temporel non stabilisé qui témoigne des ambiguïtés d'une professionnalisation inachevée ", DREES, Dossiers solidarité santé, $\mathrm{n}^{\circ} 30$.

Bouvier T., Pelvillain N., Santelmann P. (2010) : "Services à la personne : renforcer l'encadrement intermédiaire pour accroître l'efficacité et l'attractivité des métiers ", Formation Emploi, $\mathrm{n}^{\circ} 112$.

Burnod G., Chenu A. (2001), «Employés non qualifiés, une proposition d'aménagement de la nomenclature des catégories socioprofessionnelles ", Travail et Emploi, nº 86.

12 Le souci d'équilibre des comptes publics, et notamment de ceux des conseils généraux, chefs de file des politiques d'action sociale, pourrait donner la tentation de comprimer le coût par bénéficiaire de l'aide aux personnes fragiles en freinant le mouvement de qualification engagé jusque-là. 
Causse L., Fournier C., Labruyère C. (1998), Les aides à domicile, des emplois en plein remue-ménage, Syros, Paris.

CERC (2008), Les services à la personne, rapport $n^{\circ} 8$.

Charlon-Dubar E., Dubar C., Engrand S., Feutrie M., Gadrey N., Vermelle M.C. (1990), "Le salarié confronté à l'offre de formation. Trajectoire personnelle, identité professionnelle et logique d'entreprise ", Travail et Emploi, n ${ }^{\circ} 44$.

Charpin J.-M., Tlili C. (2011), Perspectives démographiques et financières de la dépendance, Ministère des Solidarités et de la Cohésion Sociale, Rapport du groupe de travail $\mathrm{n}^{\circ} 2$ dans le cadre du débat de la dépendance.

DARES (2011), Les familles professionnelles - portraits statistiques 1982-2009.

Devetter F.-X., Fraise L., Gardin L., Gounouf M.-F., Jany-Catrice F., Ribault T. (2008), L'aide à domicile face aux services à la personne-Mutations, confusions, paradoxes, Rapport pour la DIIESES, Clerse-Crida-Réseau 21, mars, 308p.

Devetter F.-X., Horn F., Jany-Catrice F. (2011), «Interprétations localement différenciées d'une politique nationale : l'exemple des services à la personne ", Revue Française des Affaires Sociales, $\mathrm{n}^{\circ}$ 4, octobre-décembre, pp. 134-157.

Doniol-Shaw G., Lada E., Dussuet A. (2007), Les parcours professionnels des femmes dans les métiers de l'aide à la personne. Leviers et freins à la qualification et à la promotion, rapport de recherche LATTS.

Doniol-Shaw G. (2011), "Quels diplômes pour le secteur de l'aide à la personne ? ", Formation Emploi, n ${ }^{\circ} 115$, pp. 51-65.

Ennuyer B. (2009), Repenser le maintien à domicile, Éditions Dunod, 270 p.

Fournier C., 2004, « La formation tout au long de la vie à l'épreuve du genre ", Ville-ÉcoleIntégration-Enjeux, $\mathrm{n}^{\circ} 136$, mars.

Fourcade B., Ourtau M. (2004), "Les employés non qualifiés : entre rationalisation et professionnalisation ", in Le travail non qualifié, Méda, Vennat (eds), pp. 310-322.

Fournier C. (2006), "Les besoins de formation non satisfaits des salariés au prisme des catégories sociales ", Formation Emploi n ${ }^{\circ}$ 95, pp. 25-39.

Fournier C. (2009), "Concilier vie familiale et formation continue, une affaire de femmes ", Céreq-Bref n 262.

Giret J.-F., Lemistre P. (2004), « Le déclassement à l'embauche des jeunes : vers un changement de la valeur des diplômes? ", Cahiers économiques de Bruxelles, vol. 47, n 3/4, pp. 483-503.

Giret J.-F., Nauze-Fichet E., Tomasini M. (2006), « Le déclassement des jeunes 
sur le marché du travail », Données sociales, pp. 307-314.

Jany-Catrice F., Puissant E., Ribault T. (2009), « Associations d'aide à domicile : pluralité des héritages, pluralité des professionnalités ", Formation Emploi n 107, pp. 77-91.

Jolly C., Liégey M., Passet O. (2012), « Les secteurs de la nouvelle croissance : une projection à l'horizon 2030 », Centre d'analyse stratégique, Rapports \& Documents, janvier.

Lada E. (2011), «Les recompositions du travail d'aide à domicile en France ", Formation Emploi, $\mathrm{n}^{\circ} 115$, pp. 9-22.

Lainé F., Omalek L. (2012), "Les métiers en 2020 : progression et féminisation des emplois les plus qualifiés ; dynamisme des métiers d'aide et de soins aux personnes ", DARES et Centre d'analyse stratégique, Dares Analyses, $\mathrm{n}^{\circ} 022$, mars.

Laforge G. (2003), Les emplois familiaux - Réflexions sur une politique de l'emploi, rapport de thèse de droit public, université de Nantes, Laboratoire Droit et Changement Social, $735 \mathrm{p}$.

Laroque (1962), Rapport de la Commission d'étude des problèmes de la vieillesse, dit rapport Laroque.

Marquier R. (2010a), «Échantillonnage, apurements et redressement de la non-réponse dans l'enquête IAD - enquête auprès des intervenants au domicile des personnes fragilisées (2008) ", DREES, Document de travail série Sources et Méthodes, n 11, juin.

Marquier R. (2010b), "Les intervenantes au domicile des personnes fragilisées en 2008 ", DREES, Études et Résultats, n 728 , juin.

Rose J. (2012), Qu'est-ce que le travail non qualifié?, La Dispute. 